\title{
Silentiaires fin-de-siècle : émergence et épuisement d'un motif poétique
}

Se taire ou parler ? Cette énième variation sur la tirade d'Hamlet, ou plutôt ce qui en surnage dans la culture populaire, agitait déjà La Bruyère qui estimait que l'on vient toujours trop tard, quand tout a déjà été dit. Si le romantisme coïncide avec l'émergence de l'individualisme en Occident et pose la question de l'originalité en art, la charnière qui relie la fin du XIX ${ }^{\mathrm{e}}$ siècle avec le début du $\mathrm{XX}^{\mathrm{e}}$ fut le théâtre d'un vertige ontologique dont Mallarmé constitue sans doute l'acteur le plus marquant, et qui menace de déboucher sur une véritable négation de tout geste artistique ${ }^{1}$. Henri Garric, dans la somme qu'il a récemment consacrée aux liens qu'entretiennent la création artistique et le silence, rappelle le défi que se lance Coleridge à la suite de la lecture qu'il fait de Plotin et qui tient tout entier dans l'oxymore de la parole silencieuse :

Il va lui falloir inventer un taire actif ou à l'inverse une expression qui se définirait paradoxalement comme silencieuse. C'est ce paradoxe qui se trouve au centre de l'esthétique littéraire aux $\mathrm{XIX}^{\mathrm{e}}$ et $\mathrm{XX}^{\mathrm{e}}$ siècle [sic]. La littérature ne cherche pas à le résoudre : elle le creuse au contraire en faisant de tout objet silencieux son sujet, comme pour dire qu'à force de répéter les lieux communs du silence, elle fera du silence son bien (Garric, 2015 : 91).

Le symbolisme fut lourd de cet enjeu, depuis l'édifiant constat auquel le Petit Glossaire pour servir à l'intelligence des auteurs décadents et symbolistes du prétendu Jacques Plowert contribue en répertoriant la forme adjectivale silent dérivée de silencieux jusqu'à l'entrée silence des 100 mots du symbolisme de Jean-Pierre Bertrand et Paul Aron qui précise qu'il fut " partie intégrante de l'imaginaire symboliste ». Se

Antoine Piantoni - docteur, chercheur associé au Labex Obvil de l’Université Paris-Sorbonne. Adresse pour correspondance : Maison de la Recherche, 28 rue Serpente, 75006 Paris ; email : antoine. piantoni@yahoo.fr

1. On rappellera pour mémoire la « crise de Tournon » qui ébranle le poète en 1866-1869 et se cristallise notamment dans la rédaction d'Hérodiade : "Hérodiade est, elle-même, à travers sa virginité, le symbole du Néant, sur la forme de la blancheur et dans son silence énigmatique » (Drigo Agostinho, 2009 : 2). 
structure en l'espace de quelques décennies une forme de révérence à l'égard du silence qui confère à l'écrivain en général et au poète en particulier le hiératisme d'un silentiaire, officier du Bas Empire romain chargé de faire régner l'ordre et le silence dans les réceptions et notamment en présence de l'empereur ${ }^{2}$. Ce que H. Garric analyse comme un défi aporétique, Daniel Grojnowski l’a rapproché de la mystification, notamment en prenant comme support de sa réflexion la nouvelle de Villiers de l'Isle-Adam intitulée " Le Secret de l'ancienne musique » : dans ce conte, le dernier musicien spécialiste du chapeau chinois, "instrument militaire aujourd'hui tombé en désuétude », est tiré de sa réclusion pour interpréter une partition d'un compositeur allemand qu'on devine sans peine être Wagner. Le morceau n'est composé que de silences dont l'" exécution, toujours sobre, mais pleine de nuances, était d'un style si châtié, d'un rendu si pur, que, chose étrange ! il semblait, par moments, qu'on l'entendait! » (Villiers de L'Isle-Adam, 1986 : 641). Le déraillement du sens participe d'une maïeutique de l'effroi, car « son secret réside dans la relation des lecteurs à leur propre "partition", c'est-à-dire au récit qu'ils viennent de lire »(Grojnowski, 2005 : 449). Nous souhaiterions justement mesurer cet écart qui va s'augmentant entre un thème qui s'abreuve aux sources d'un certain mysticisme symboliste et sa progressive dénaturation qui le fait basculer dans une fascination proche du nihilisme jusque dans l'humour ravageur.

\section{Sous le signe d'Harpocrate}

La peinture ne fut pas avare de représentations de cette divinité égyptienne hellénisée qu'on songe au pastel de Fernand Khnopff, intitulé Du Silence (1890) ou bien à l'huile Silence (1911) d'Odilon Redon, donnant tous deux à voir une figure un doigt à la bouche. De fait, cette incarnation du silence a fasciné les symbolistes de la fin du siècle par ses liens avec les arcanes ésotériques, incitant certains d'entre eux à utiliser le motif du silence comme une métaphore métapoétique. Ainsi, Francis Vielé-Griffin qui, à partir d'un fragment hagiographique de la vie de sainte Gudule, construit une fable mystique non dénuée d'un certain panthéisme, dans laquelle une abbesse noue un lien privilégié avec un ours réfugié dans son abbaye :

Hors la Prière où l’on parle à Dieu

L'on ne parlait pas dans ce calme lieu.

Et l'ours, ainsi, entendait l'abbesse

Qui parlait des yeux ou d'une caresse,

2. Plusieurs figures de la fin de l'Antiquité et des débuts du Moyen Age, religieuses et laïques, portèrent le surnom de silentiaire : par exemple saint Jean le Silentiaire, ou l'Hésychaste (« celui qui est en paix, qui garde le silence »), moine de la fin du Ve siècle, ou bien Paul le Silentiaire, poète byzantin du $\mathrm{VI}^{\mathrm{e}}$ siècle qui assura la charge de silentiaire sous Justinien. 
Et l'abbesse, dans le silence, savait

Lire en les yeux de l'ours ce qu'ils savaient rêver ;

Ils se disaient ainsi des choses qu'on ignore

Parce que la parole est trop sonore,

Que la vie est brutale et banale par elle

Et que le silence est la langue éternelle

Où les hautes choses et les choses saintes

Flottent de rêve en rêve, de pensée en pensée,

Où l'ample idée s'éveloppe sans la feinte

Des pauvres mots de nos lèvres osées :

Le silence ami où la Belle et la Bête

Parlaient le rêve du poète (Vielé-Griffin, 1893 : 45-46).

Vielé-Griffin tire délibérément son argument du côté du conte dans l'avant-dernier vers en y adjoignant cependant une dimension supplémentaire dans le dernier, ce "rêve du poète ", devenu, par la vertu de la transitivité du verbe " parlaient », idiome insaisissable au bord de l'ineffable. Le poète suggère à travers l'expérience mystique une possibilité de renouer avec une langue originelle, fictive mais séduisante, que les linguistes ont traquée au XIX ${ }^{e}$ siècle, suivis en cela par les littérateurs, Mallarmé en tête. Il est significatif que ce trajet emprunte les voies impénétrables du Seigneur, car, comme l'a rappelé $\mathrm{H}$. Garric, la poésie mystique fonctionne elle-même sur ce principe oxymorique d'un langage coextensif au silence. Garric cite l'une des Chansons entre lầme et l’époux de Jean de la Croix qui évoque la « musique tue ${ }^{3}$ » de la nuit mystique:

Avec cet oxymore, la poésie désigne une expression qui passe par le silence, au moment même où elle invente un langage qui dépasse le langage. Elle invente une expression silencieuse tout en donnant un exemple de ce que cette expression pourrait être : un langage qui, ayant pris acte de l'imperfection du langage humaine, le défait et recompose simultanément pour tenter de s'approcher d'une expérience qui le dépasse et qui ne peut, au sens strict, être atteinte qu'en sortant de l'humain, c'est-à-dire en se plongeant dans le silence. [...] La " parole muette » à laquelle permettait de rêver l'origine des langues trouve son équivalent dans une parole poétique élaborée pour atteindre la divinité et qui, pour ce faire, brise le carcan de la langue humaine (Garric, 2015 : 97-98).

Cet effort de ressaisissement d'un noyau ontologique obscur par la parole poétique se diffuse dans nombre d'œuvres symbolistes, comme l'étrange Conte de l'Or

3. La nuit calme et heureuse

toute proche du lever de l'aurore, musique silencieuse, solitude sonore,

repos, amour, le souper qui restaure.

(Thérèse d'Avila-Jean de la Croix, 2012 : 703) 
et du Silence que Gustave Kahn dédie à Mallarmé en 1898. L'or et le silence, dont les deux cultes sont exposés au début de l'ouvrage, apparaissent comme une variation sur l'affrontement de la lumière et de l'ombre, le substrat de toute mythologie selon Mallarmé, mais également une méditation platonicienne sur l'idéalisme qui renaît en cette fin de siècle. Les premières pages contiennent un hymne au Silence, divinité dont le culte a été institué par le roi Balthazar, que nous ne citerons que partiellement :

Silence, fond unique et nécessaire de la Parole,

de la parole d'un qui se lève pour dire les mots fondamentaux, les seuls mots courts qui indiquent le culte,

le culte du silence absolu.

Silence, dieu persécuté par les tyrans et par la plèbe

Dieu massacré dès l'aube par les chariots des gravatiers

Dieu étoilé des sanguinolentes blessures de la parole impatiente et drue et ambitieuse que les pauvres dénomment le Verbe

$[\ldots]$

Tu tiens la coupe étrange et dernière,

Le philtre de merveille de tous les jours contre les dieux d'activité, contre les machines défiées de gloire ou de terreur par des foules affamées,

Tu la tiens sans jamais la tendre et il faut gravir les aspérités de toutes les routes sombres des mortelles échéances, pour, dépourvu d'orgueil, y venir tenter

la chance suprême de bonheur, en ton incarnation, Silence (Kahn, $1898: 13-14)$.

Monde des idées plus fécond que celui des créatures physiques, le silence est constitutif du geste artistique, vide que viennent combler « les personnages de [l'] âme de Balthazar » dans une dramaturgie immatérielle et autarcique. La litanie du silence se déploie à la manière d'un ouroboros, perpétuel retour au point d'origine, du néant au néant. Ces deux exemples peuvent être envisagés comme la pointe extrême de ce continent mutique et contemplatif décrit de manière fragmentaire par les auteurs symbolistes.

Parallèlement, une forme de déplétion ou d'affadissement se fait sentir dans des textes qui traitent le silence davantage comme un motif que comme une clef de compréhension métaphysique. Les « Chants de la matière » de Maxime Du Camp avaient dès le milieu du XIXe siècle donné droit de cité à la vie bruyante et énergique des objets comme la locomotive ou le métier à tisser; le symbolisme réserve une place d'un autre ordre, davantage méditative : le corollaire des intérieurs reconstitués dans nombre de poèmes se trouve dans cette vie silencieuse des objets. Le Règne du silence (1891) de Georges Rodenbach offre une plongée dans cette atmosphère suggestive, très verlainienne, qui peut permettre, à la faveur d'une expérience sensorielle et mé- 
taphysique, une écoute inouïe de la vie intérieure. C’est précisément ce qu'explique un critique assez sévère avec le poète belge :

Un jour, vous êtes demeuré longtemps, dans une chambre reléguée, environnée de silence ; environnée, c'est-à-dire que, d’abord, ce silence était partout autour de vous, et que vous vous y baigniez comme l'oiseau dans l'air ou le poisson dans l'eau ; environné, puis, peu à peu, pénétré par ce silence qui s'insinuait en vous, dans votre cœur, dans votre cerveau, et y éteignait tous les bruits de la vie, y suspendait tous les mouvements de l'âme, si bien que, au bout de quelques heures, l'endosmose a été complète, l'équilibre s'est établi entre le silence extérieur et le silence intérieur, et vous vous êtes senti en parfaite communions avec le milieu ambiant, avec l'atmosphère.

À ce moment-là, - et pas avant, - vous êtes dans l'état physiologique et psychologique voulu pour entendre les « voix du silence » $[\ldots]$.

Vous souriez ? Vous avez tort. Il ne faut pas sourire. Cela est très sérieux. Rien n'est plus sérieux que le langage du silence. On vous explique et on vous répète, pour que vous le compreniez bien, que les sens d'un poète affinés dans un bain de solitude et de silence perçoivent des harmonies nouvelles (Carrara, 1915 : 1218).

Le critique tourne ici en dérision les prétentions décadentistes d'atteindre à une sensibilité exacerbée, et met au jour par là même un ensemble de lieux communs qui définissent un rapport qu'on pourrait qualifier de suspect avec le silence : s'agit-il d'une expérience mystique ou bien le poète se satisfait-il d'un décorum qui le plonge dans une réceptivité feinte ? Un extrait d'une des pièces de la section " Du silence " trahit ce vertige provoqué : le poète y évoque sa fraternité avec les âmes simples et humbles qu'on devine être celle des moniales :

Oh ! vous mes Sours - car c'est ce cher nom que l'Église

M'enseigne à vous donner, sœurs pleines de douceurs,

Dans ce halo de linge où le front s'angélise,

Oh! vous qui mêtes plus que pour d’autres des sœurs

Chastes dans votre robe à plis qui se balance,

O vous mes sœurs en Notre Mère, le Silence ! (Rodenbach, $1891: 204$ )

La personnification féminine a de quoi surprendre : c'est qu'elle traduit imparfaitement une sorte de lénification que l'on serait tenté de relire comme un commentaire métatextuel sur la dévitalisation du verbe poétique. Un autre passage est plus explicite sur l'avortement de la vocation artistique qui sous-tend le recueil :

Et le soir, sur les eaux doucement remuées,

Ces cygnes imprévus, venant on ne sait d'où,

Dans un chemin lacté d'astres et de nuées

Mangent des fleurs de lune en allongeant le cou. 
Or ces cygnes, ce sont des âmes de naguères

Qui n'ont vécu qu'à peine et renaîtront plus tard,

Poètes s'apprenant aux silences de l'Art,

Qui s'épurent encore en ces blancs sanctuaires,

Poètes décédés enfants, sans avoir pu

Fleurir avec des pleurs une gloire et des nimbes,

Ames qui reprendront leur Euvre interrompu

Et demeurent dans ces canaux comme en des Limbes! (Rodenbach, 1891 : 217-218)

Comme le prophétisait Nerval dans ses "Vers dorés ", "À la matière même un verbe est attaché ", mais cette parole étrangère à l'humain laisse ce dernier face à l'énigme de la matière qui ne peut faire l'objet que de projections anthropomorphiques. Maurice Rollinat, le chantre débridé des Névroses, voit dans le silence des vertus curatives dont l'explication doit demeurer mystérieuse :

Le silence est l'âme des choses

Qui veulent garder leur secret.

Il s'en va quand le jour paraît,

Et revient dans les couchants roses.

Il guérit des longues névroses,

De la rancune et du regret.

Le silence est l'âme des choses

Qui veulent garder leur secret.

À tous les parterres de roses

Il préfère un coin de forêt

Où la lune au rayon discret

Frémit dans les arbres moroses :

Le silence est l'âme des choses (Rollinat, $1885: 19$ ).

Le vers surnuméraire, qui transforme le quatrain final en quintil, contribue à une forme d'ambiguïté : de quel phénomène inaccessible le silence est-il l'écho paradoxal ? De quel envers bruyant est-il l'avers insaisissable ? Sur un mode plus léger, d'aucuns diront plus mièvre, Albert Samain construit une suite de tercets à rime unique qui, d'une situation banale, celle d'un couple réuni avant l'étreinte ${ }^{4}$, bascule progressivement vers la tentation du suicide commun. Cette réécriture probable de « La Mort des amants » propose une variation mineure et se clôt sur un phénomène similaire à celui relevé dans le poème de Rollinat :

4. Rodenbach compose également une rêverie qui dévide le mythe platonicien de l'androgyne : «Et langoureusement la clarté se retire ;/ Douceur ! ne plus se voir distincts ! Nêtre plus qu'un !/ Silence ! deux senteurs en un même parfum :/ Penser la même chose et ne pas se le dire » (Rodenbach, 1891 : 186). 
Oh ! s'en aller sans violence,

S'évanouir sans qu'on y pense

D’une suprême défaillance...

Silence !... Silence !... Silence!... (Samain, $1924: 207)^{5}$

À nouveau, la formulation paradoxale du silence passe par sa profération, le substantif portant sa propre charge d'annulation ${ }^{6}$. Le texte entier acquiert du même coup une valeur propitiatoire, renouant avec la dimension mystique de poèmes comme ceux de Kahn ou Vielé-Griffin, mais selon un principe d'affadissement ou de déperdition qui exhibe le prétexte thématique pour mieux conjurer le « taire actif » dont parle H. Garric. La conjonction de la mort et du silence n'est en rien exceptionnelle et sacrifie bien au lieu commun, mais elle matérialise un appel au néant, un silence dans et du texte, pour reprendre la formule de Jean de Palacio.

Pour ce dernier, lère de crises, qui voit se développer les esthétiques symbolistes, est propice à un dérèglement des formes qui matérialise une sorte de décadence nihiliste. Le silence du texte se perçoit dans l'expression d'un épuisement, notamment des formes fixes à l'architecture codifiée - comme le rondeau ou le sonnet. Jean de Palacio distingue d'ailleurs trois phases « de la pulvérisation et de l'atomisation du texte » : le choix de l'octosyllabe au détriment de l'alexandrin, l'avènement du monosyllabe et enfin le sonnet «nihiliste » ou "pseudo-sonnet » dont la manifestation la plus exaspérée et iconoclaste est le sonnet blanc que l'on trouve chez Georges Fourest avec son «Pseudo-sonnet que les amateurs de plaisanterie facile proclameront le plus beau du recueil » entièrement constitué de croix ou bien chez Marius Boisson, dans sa forme la plus radicale de page blanche titrée "Sonnet fantastique». Cet évidement ultime, qui pose la question de la mystification que l'on trouvait en filigrane du conte de Villiers de l'Isle-Adam, interroge corollairement la pertinence de la tentative poétique : « $\mathrm{Ne}$ rien dire et dire quelque chose revient donc au même. Le texte parle en se taisant, du fond d'une forme vide dont il ne subsiste que le contour» (Palacio, $2003: 81$ ).

\section{Rien n'est plus sérieux que le langage du silence}

Le stade terminal de cette exploitation du silence - comme moteur esthétique - se situe dans la parodie pure et simple. Nous prendrons, comme cas représentatif, un canular échafaudé par Jean-Marc Bernard, poète et critique proche de l’Action française qui renia sa propre jeunesse symboliste dans les quinze premières an-

5. On notera que ce poème, dont la date exacte de rédaction n'est pas connue, peut se lire en parallèle d'un autre poème homonyme de Francis Jammes, justement dédié à Samain, publié dans La Revue blanche en novembre 1896 et repris dans De l'Angélus de l'aube à l'angélus du soir en 1898. Le texte est une rêverie nostalgique sur les " amours des vieux temps", chers à Jammes.

6. Le poème sera tout de même mis en musique par Julien Naudy en 1910. 
nées du XXe siècle. Un entrefilet daté de février 1910 de la revue Les Guêpes, dont Bernard est l'un des animateurs, annonce la parution d'une nouvelle revue, Le Père Fouettard, sous la direction de Jean-Charles-Émile Rey, à Crest, dans la Drôme. C'est Jean-Marc Bernard lui-même qui se charge de signaler l'événement, en précisant : " Le premier fascicule publiera une lettre de Jean Royère à propos de dix vers inédits de Stéphane Mallarmé. Qu'on se le dise ! » ${ }^{7}$. Nous n’avons pas trouvé la trace de cette revue, dirigée par l'un des rédacteurs d'épigrammes des Guêpes, dont le nom a vraisemblablement servi de paravent pour certains contributeurs. Nous postulons l'hypothèse d'un canular, visant à discréditer Royère ${ }^{8}$, défenseur de l'héritage mallarméen à travers le courant néo-symboliste et directeur de La Phalange ; et Jean-Marc Bernard avoue en être au moins en partie responsable dans le numéro suivant des Guêpes :

Nous tenons à signaler, ainsi que nous l'avons promis, une lettre de Jean Royère à propos de dix vers inédits de Mallarmé. Voici les passages les plus intéressants de cette lettre :

"Vous serez bien aimable de me donner tous les renseignements possibles sur les vers de Mallarmé que vous avez et la délicate pensée de recopier à mon intention. Ces vers ne figurent pas sur les éditions complètes du poète et je ne les ai lus nulle part : ils sont certainement inédits. Il serait intéressant que vous contiez avec des détails pour nos lecteurs comment vous les avez découverts, chez qui : bref, que vous écriviez à ce propos un article que nous publierons avec les vers. Ils sont certainement très mallarméens. » ${ }^{9}$

Bernard précise que La Phalange n'a pas daigné publier les vers envoyés à Royère et produit ensuite le poème en question, avant d'ajouter : " Néanmoins, M. Royère doit joliment se repentir d'avoir envoyé cette lettre. Pensez donc : ces vers si mallarméens nétaient pas de Mallarmé, mais du "pauvre insulteur de ce classique suprême"! Me, me adsum qui feci $i^{10} ! »$. Bernard donne ici suite à la lettre qu'il avait envoyée à Royère, parue dans La Phalange du 20 septembre 1909, en réponse à une chronique de Robert de Souza publiée sous les initiales G. T. (correspondant à Georges Tornouel, pseudonyme de Souza), dans la livraison de la même revue, le 20 juillet précédent, dans laquelle ce dernier entendait mettre Bernard devant ses contradictions et le qualifiait effectivement de "pauvre insulteur de ce classique suprême que fut Stéphane Mallarmé ». Le faux est cité, sans titre :

7. « Note. Le Père Fouettard », Les Guêpes, février 1910, p. 56.

8. Le directeur de La Phalange assume, malgré lui, la fonction d'intermédiaire telle que l'a décrite Daniel Bilous : « En littérature du moins, la supercherie mobilise presque toujours un intermédiaire, qui sera le premier abusé et le plus facilement abusable, parce que le premier intéressé par de l'inédit : rédacteur ou comité de rédaction d'un périodique apte à le diffuser auprès du lectorat » (Bilous, 2006 : 206).

9. « Note. Le Père Fouettard et Jean Royère », Les Guêpes, mars 1910, p. 86-87.

10. Il s'agit d'une citation de Virgile (Énéide, IX, 427). 
Funèbre cette nuit présage maints désastres

Inscrits au ciel en la noire absence des astres

Pour le poète seul agonisant ici ;

Car la stérilité de son morne souci

À jamais le retient esclave tributaire

Du Silence fatal du Verbe qu'il dût taire ;

Aussi bien si d'un mot vierge ensemble et subtil

Quelque jour, ignoré hélas! le rompait-il,

Pur Il s'exhalerait parmi le soir insigne

Et pareil à la mort sur l'étang d'aucun cygne.

Bernard reprend le poème en le modifiant substantiellement dans son propre recueil Sub Tegmine Fagi, en 1913, sous le titre «Silence » avec, en épigraphe, un extrait de la lettre de Royère :

«Ces vers ne figurent pas sur les éditions complètes du poète (S. Mallarmé) et je ne les ai lus nulle part : ils sont certainement inédits. »

Jean Royère, lettre du 14 décembre 1909.

Funèbre cette nuit présage maints désastres

Inscrits au ciel en la noire absence des astres

Pour le poète seul agonisant ici ;

Silence ! et que retient ton stérile souci;

Car pour l'éternité le voici tributaire

Du Verbe que malgré son cœur il a dû taire.

Aussi bien si d'un mot vierge ensemble et subtil

Quelque jour, ignoré hélas! te rompait-il,

Pur tu t'exhalerais parmi le soir insigne

Et pareil à la mort sur l'étang d’aucun cygne (Bernard, 1913: 121-122).

Faire figurer le poème dans le recueil signifie qu'il est considéré comme son auteur, comme partie intégrante de son œuvre de poète. Le choix de conserver en épigraphe un extrait de la lettre de Royère témoigne en revanche d'un souci de rappeler le succès de la supercherie. Le poète dauphinois montre qu'il est capable d'utiliser ses talents pour piéger les lecteurs, et surtout le lecteur exceptionnel que devrait être Royère. Bernard démontre ainsi qu'il comprend Mallarmé, par l'exemple, puisqu'il est capable de produire $d u$ Mallarmé suffisamment convaincant pour flouer un de ses thuriféraires. Dans ce cas précis, le faux est d'un usage polémique et exemplaire, à la manière de La Chasse spirituelle d'Akakia-Viala et Nicolas Bataille, composé en 1949 dans le but de prouver leur compréhension de l'œuvre de Rimbaud après le tollé de leur adaptation théâtrale d'Une saison en enfer. Nous abordons aux frontières floues entre pastiche et forgerie, car le phénomène se déroule en deux temps : diffusion du texte qui mène ou non à une méprise puis révé- 
lation du procédé qui discrédite la cible en même temps qu'il démontre l'adresse du rédacteur ${ }^{11}$. Dans la reprise en volume, Bernard ne se contente pas de mettre en lumière ce qui était resté à l'état d'entrefilet dans une petite revue. Le texte est suivi d'un florilège de quatre citations, chacune occupant une pleine page comme une épigraphe suivant le poème. Ces (très) brèves citations ont en commun d'évoquer le silence ; on trouve de Rimbaud : "C'est trop beau ! trop ! Gardons notre silence. », de Mallarmé : "Musicienne du silence. ", de Verlaine : "Silence, silence !» et de Samain : «Silence !... silence !... Silence !... $»^{12}$. Comment interpréter ce choix dont la mise en page suggère une mise en scène du silence (Bernard aurait pu faire figurer ces citations de manière classique en tête de son poème) ? Comment également comprendre ces citations qui ont perdu tout caractère exemplaire et sont dépourvues de toute autorité ${ }^{13}$ ? Bernard s'inscrit contre l'esthétique mallarméenne, ou du moins contre une interprétation de celle-ci ${ }^{14}$. Il nous semble que la clef de compréhension réside justement dans cette disposition : en mettant à la suite de son poème-supercherie des pages vides qui ne contiennent que la mention ténue du silence, à travers d'éminents représentants du symbolisme, Bernard met en place une équivalence figurale ; son texte, intitulé " Silence " pour l'occasion, ne débouche sur rien, non plus que les citations. Le tout ne donne accès qu'à une béance, n'a pour objet que le vide qu'il produit. Une dimension supplémentaire s'ajoute alors au faux de Bernard; ce dernier ne le considère pas comme une preuve de son habileté mais semble le renvoyer à une autre supercherie, celle des néosymbolistes qui perpétuent l'erreur de Mallarmé.

Dans les parages du symbolisme, le silence demeure fécond, tant comme motif que comme outil herméneutique, mais cet instrument est à double tranchant : fine est la frontière entre mystique authentique et prétexte à un vertige contrôlé. Le paradoxe du silence, qui se donne à entendre dans et par la lecture, condamne l'inef-

11. On peut parler de mystification au sens où l'entend Jean-François Jeandillou : «Par différence avec la contrefaçon ou l'usage de faux juridiquement punissables, la tromperie ne se fonde pas sur un véritable abus de confiance, car elle n'est conçue que pour être prise en défaut : d'ailleurs, c'est au moment de la démystification que le plaisir du mystificateur atteint son point culminant » (Jeandillou, 1989 : 490).

12. Ces citations sont respectivement extraites de « Bruxelles » (Derniers vers), « Sainte » (Poésies), «Un grand sommeil noir.. » (Sagesse) et «Silence !... » (Au Jardin de l'Infante). Ce dispositif n’a pas été conservé dans l'édition posthume des œuvres de Jean-Marc Bernard parue en 1923.

13. Faut-il lire cette accumulation comme une portée musicale similaire à celle que tente d'interpréter le protagoniste du Secret de l'Ancienne Musique de Villiers de L'Isle-Adam ?

14. «La poésie réalise ainsi une synthèse du silence et de la parole - silence par rapport à l'objet tu, parole par rapport aux allusions qui l'indiquent. De l'objet tu à l'objet absent, les frontières sont indécises. En des sonnets comme Une dentelle s'abolit, Mes bouquins refermés, le poète, au lieu de taire l'objet, trouvera dans l'absence formellement indiquée un équivalent esthétique du silence » (Thibaudet, 1912 : 113). Le motif du cygne pourrait d'ailleurs renvoyer à cette observation du même Thibaudet : «Le silence pour lui n'est pas un vide, mais une corde tendue, une capacité indéfinie de musique, prise dans le gel ainsi que le Cygne, et qui ne peut bruire » (ibid. : 138). 
fable à basculer dans l'inarticulé du néant, oblitérant par la même occasion le sérieux d'une entreprise qui n'est rien moins que la justification de la poésie. Le recours au silence en poésie est comparable à l'usage qui peut en être fait en société : «Le silencieux porte à son insu l'énigme de la parole absente et laisse miroiter l'horreur d'une société sans langage » (Le Breton, 2015 : 64). Le silence risque dès lors d'être perçu comme le refuge des hypocrites et la matrice d'une mascarade que les plus vindicatifs, à l'instar de Jean-Marc Bernard, rêveront si ce n'est de détruire, du moins d'en mettre l'imposture en plein jour.

\section{BIBLIOGRAPHIE}

Aron P. \& Bertrand J.-P. 2011. Les 100 mots du symbolisme. Paris. PUF. Coll. « Que sais-je ?». Bernard J.-M. 1913. Sub Tegmine Fagi. Paris. Éditions du Temps présent.

Bilous D. 2006. Mallarmé aux miroirs. In Bilous D. Mallarmé, et après? Fortunes d’une ceuvre. Paris. Noésis Éditions. 197-228.

Carrara J. 1915. Croquis belges IV. La Guerre mondiale 153. 1217-1218.

Drigo Agostinho L. 2009. De la page blanche à la musique : Mallarmé et l'écriture du silence. In Logosphère 5. Écritures du silence. 1-12.

Garric H. 2015. Parole muette, récit burlesque. Les Expressions silencieuses aux XIXe-XXe siècles. Paris. Classiques Garnier. Coll. « Perspectives comparatistes. Série Modernités et avantgardes ».

Grojnowski D. 2005. Mystification et mystère. Poétique 144. 443-455.

Jeandillou J.-F. 1989. Supercheries littéraires. La vie et l'œeuvre des auteurs supposés. Paris. Usher.

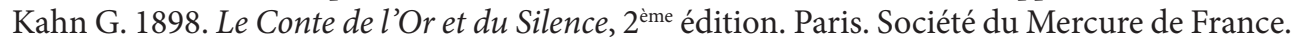
Le Breton D. 2015. Du silence. Paris. Métailié. Coll. « Suite essais ».

Palacio (de) J. 2003. Le Silence du texte. Poétique de la décadence. Louvain-Paris-Dudley. Peeters.

Rodenbach G. 1891. Le Règne du silence. Paris. Bibliothèque Charpentier.

Rollinat M. 1885. Les Névroses, $6^{\text {ème }}$ mille. Paris. G. Charpentier et $C^{\text {ie }}$.

Samain A. 1924. Euvres, t. I. Paris. Mercure de France.

Thérèse d'Avila. Jean de la Croix. 2012. Euvres. Éd. Jean Canavaggio, avec la collaboration de Claude Allaigre, Jacques Ancet \& Joseph Pérez. Paris. Gallimard. Coll. « Bibliothèque de la Pléiade».

Thibaudet A. 1912. La Poésie de Stéphane Mallarmé. Paris. Éditions de La NRf.

Vielé-Griffin F. 1893. La Chevauchée d'Yeldis et autres poèmes. Paris. Léon Vanier.

Villiers de l'Isle-Adam A. 1986. CEuvres complètes, t. I. Éd. Alan Raitt \& Pierre-Georges Castex, avec la collaboration de Jean-Marie Bellefroid. Paris. Gallimard. Coll. "Bibliothèque de la Pléiade ». 
Fin-de-siècle Silencers: rise and fall of a poetical motive

ABSTRACT: The parallel urge of introspection and meditation which comes with the evocation of silence acts as landmark for a whole current of fin de siecle literature. The symbolist era gives the impression that its actors were obsessed with silence. Symbolist poetry took possession of silence as a way to encapsulate the ineffability of feelings and the various states of mind it explored, leaning towards an aesthetical mysticism. Yet that tendency soon turned into a form of cliché that opened a path towards parody, silence becoming a stigma of the ideological shortcomings of symbolism in the eye of its detractors. The attempt to seize the silence led to a harsh confrontation with void.

Keywords: fin-de-siècle, symbolism, mysticism, parody, silence. 